\title{
Barotrauma after Manual Ventilation in a Patient with Life-Threatening Massive Hemoptysis
}

\author{
Hea Yon Lee, M.D. ${ }^{1}$, Yu Young Joo, M.D. ${ }^{2}$, Young Seung Oh, M.D. ${ }^{2}$, Yoo Rim Seo, M.D. ${ }^{2}$, Hyon Soo Joo, M.D. ${ }^{2}$, \\ Seok Chan Kim, M.D., Ph.D. ${ }^{1}$, and Chin Kook Rhee, M.D., Ph.D. ${ }^{1}$
}

'Division of Pulmonary Medicine, Department of Internal Medicine, Seoul St. Mary's Hospital, The Catholic University of Korea, Seoul; ${ }^{2}$ Department of Internal Medicine,

The Catholic University of Korea, Seoul, Korea

A 36-year-old female patient with aplastic anemia developed massive hemoptysis and was placed on ventilator support. However, airway obstruction by blood clots triggered desaturation and ventilator malfunction. Manual ventilation was initiated to improve oxygenation, and emergency flexible bronchoscopy was performed to clear the airway. Nevertheless, the patient developed extensive subcutaneous emphysema, pneumothorax, and pneumomediastinum.

Key Words: airway obstruction; barotrauma; hemoptysis; ventilation.

Barotrauma is a potentially lethal complication of positive-pressure ventilation. A high airway pressure is an important feature of the pathophysiology of barotrauma.[1] The risk of excessive airway pressure is even greater during manual ventilation of an intubated patient, because air leakage is not possible.

Airway occlusion by blood clots is fatal in cases of massive hemoptysis and is a significant risk factor for increased airway pressure in mechanically ventilated patients. Here, we report a case of extensive subcutaneous emphysema, pneumothorax, and pneumomediastinum following manual ventilation of a patient who had an airway obstruction caused by massive hemoptysis.

\section{Case Report}

A 36 year old female was admitted with a 5-day history of hemoptysis and dyspnea. She had been diagnosed with aplastic anemia at 12 years of age and had received a bone marrow transplant 5 months prior. She subsequently suffered severe graft failure and refractory pancytopenia.

On admission, her initial vital signs were: blood pressure 150/100 mmHg, heart rate 152 beats/min, respiratory rate 34 beats/

Received on April 9, 2015 Revised on May 10, 2015

Accepted on May 14, 2015

Correspondence to: Chin Kook Rhee, Division of Pulmonary Medicine, Department of Internal Medicine, Seoul St. Mary's Hospital, The Catholic University of Korea, 222 Banpo-daero, Seocho-Gu, Seoul 06591, Korea

Tel: +82-2-2258-6369, Fax: +82-2-599-3589

E-mail: chinkook@catholic.ac.kr

*There are no financial or other issues that might lead to conflict of interest. min, body temperature $36.6^{\circ} \mathrm{C}$, and an oxygen saturation level of $60 \%$ in room air. Initial arterial blood gas analysis revealed a $\mathrm{pH}$ of 7.277 , a $\mathrm{pCO}_{2}$ of $49.9 \mathrm{mmHg}$, a $\mathrm{pO}_{2}$ of $47.8 \mathrm{mmHg}$, and an $\mathrm{SpO}_{2}$ of $76.6 \%$. Her white blood cell count was 3,460/ $\mathrm{mL}$, the hemoglobin level was $7.3 \mathrm{mg} / \mathrm{dL}$, and the platelet count was $13,000 / \mathrm{mm}^{3}$. An initial chest X-ray revealed atelectasis in the left lung field (Fig. 1). Intubation was per- 


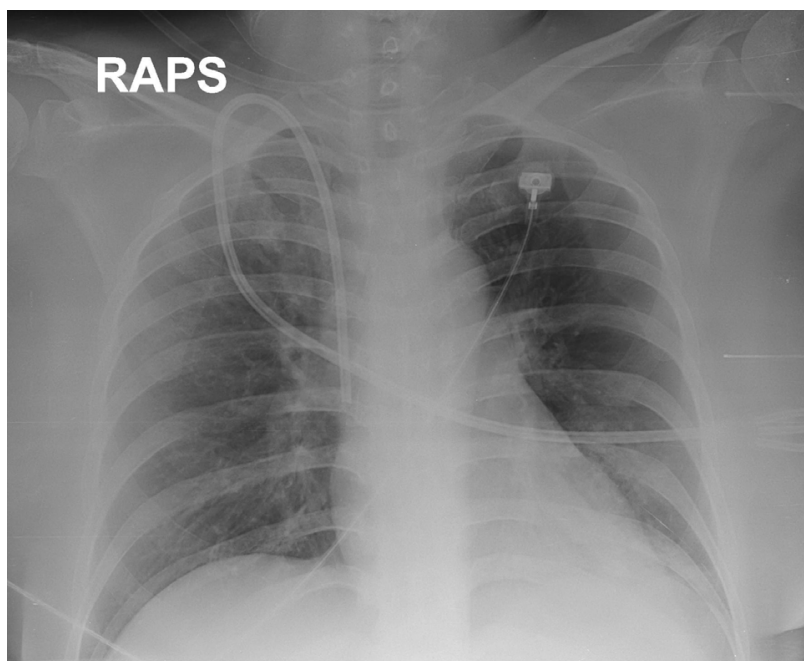

Fig. 1. A chest radiograph reveals a short, thick, subsegmental atelectasis in the lingular division of the left lung. A Hickman catheter was inserted into the superior vena cava via the right internal jugular vein. RAPS: right anterior posterior supine. formed immediately but bleeding through the endotracheal tube (ETT) continued to be significant. Suction was applied to prevent asphyxiation; about $300 \mathrm{~mL}$ blood was removed. Mechanical ventilation was applied in pressure-controlled mode with a peak pressure of $26 \mathrm{~cm} \mathrm{H}_{2} \mathrm{O}$, a positive endexpiratory pressure (PEEP) of $6 \mathrm{~cm} \mathrm{H}_{2} \mathrm{O}$, a respiratory rate of 16 breaths $/ \mathrm{min}$, and an inspired oxygen fraction $\left(\mathrm{FiO}_{2}\right)$ of 1.0. Tranexamic acid was injected intravenously and packed red cells were transfused, followed by administration of tranexamic acid and epinephrine through the ETT to seek to control the hemoptysis.

After $30 \mathrm{~min}$, the ventilator "high airway pressure" and "low expiratory tidal volume" alarms sounded. The $\mathrm{SpO}_{2}$ was $74 \%$ and the patient was cyanotic. Airway patency and saturation could be maintained only by manual ventilation using a self-inflated resuscitator bag. Manual ventilation was difficult, requiring considerable effort, because the
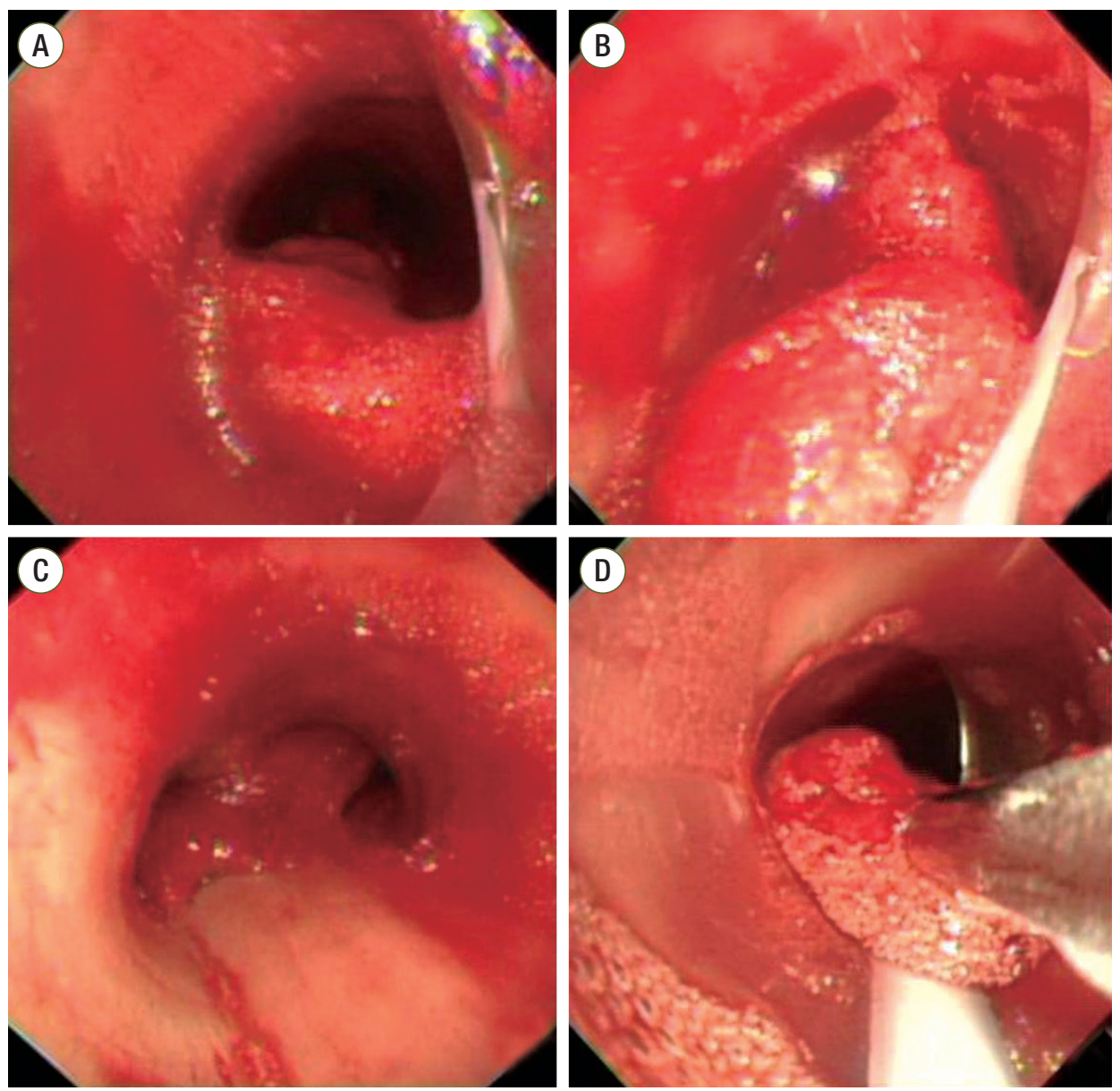

Fig. 2. Bronchoscopy reveals thick blood clots running from the distal trachea $(A, B)$ to the main carina and both bronchi $(C)$. Forceps were used to remove the clots (D). 

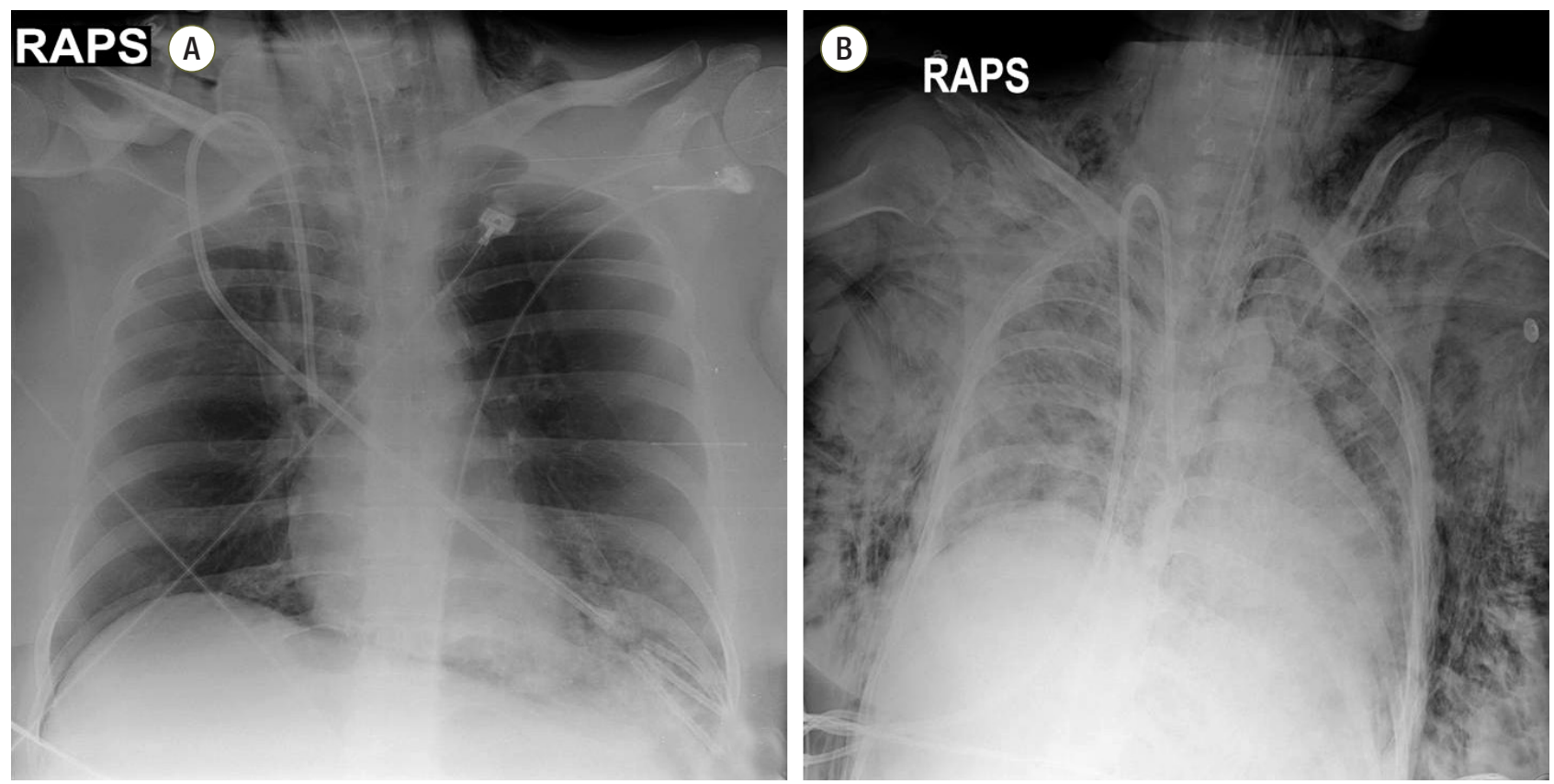

Fig. 3. (A) A post-intubation chest radiograph reveals aggravated atelectasis in the lingular division. (B) Diffuse subcutaneous emphysema is evident in the chest radiograph. RAPS: right anterior posterior supine.
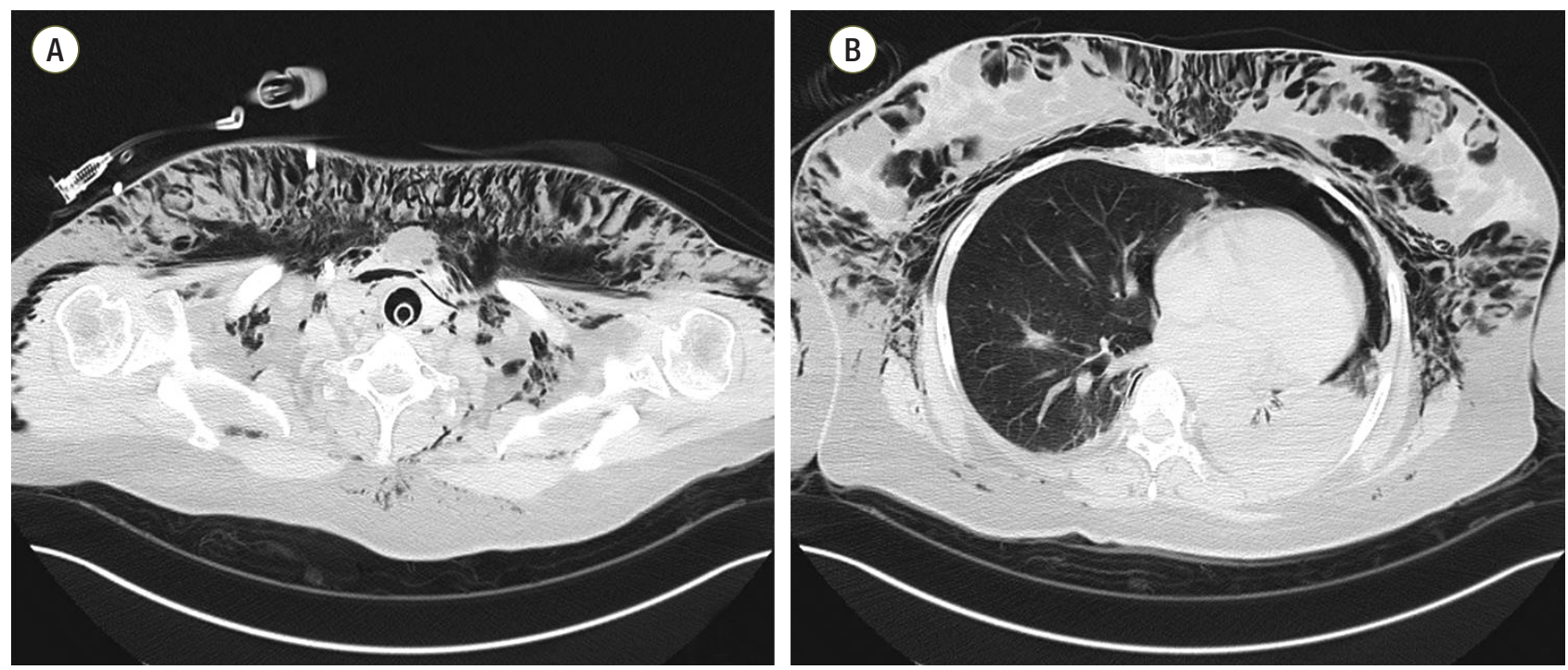

Fig. 4. (A) Chest CT reveals extensive pneumomediastinum and subcutaneous emphysema in both thoraces. (B) Moderate pneumothorax is evident in the left lung. CT: computed tomography.

trachea was partially obstructed by blood clots. Fiberoptic bronchoscopy was performed to clear the airway and locate the source of bleeding. Blood clots obstructed the airway from the distal trachea to both main bronchi. The clots in the bronchi were removed and thrombin and epinephrine were given to control the bleeding (Fig. 2). No active bleeding was noted after clot removal.
Mechanical ventilator was recommenced without any sign of airway resistance. However, the patient immediately developed extensive subcutaneous emphysema with crepitus and hyper-resonant percussion on the left abdomen. A chest X-ray (Fig. 3) and computed tomography revealed a moderate to large amount of pneumothorax and pneumomediastinum, with subcutaneous emphysema (Fig. 4). 


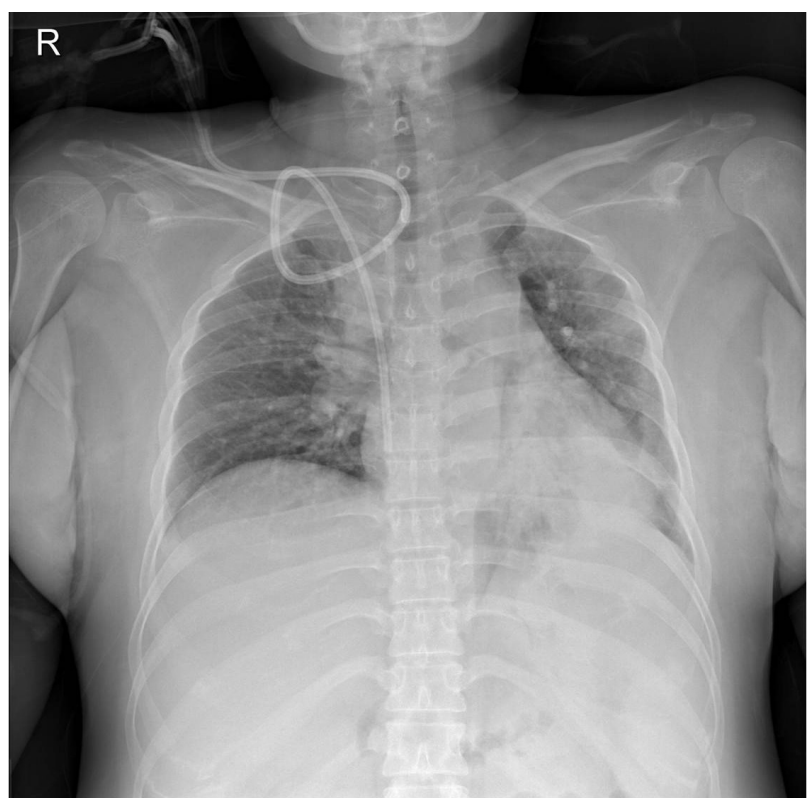

Fig. 5. Chest radiography reveals improvement of the subcutaneous emphysema and the pneumothorax of the left lung. R: right.

Because of the bleeding tendency, neither an angiocatheter nor a chest tube drain was placed. The patient's vital signs stabilized and ventilation improved in the absence of further bleeding. The pneumothorax spontaneously resolved under oxygen therapy alone; the sounds of both lungs became clear and the chest X-ray findings improved. Arterial blood gas analysis revealed a $\mathrm{pH}$ of 7.442, a $\mathrm{pCO}_{2}$ of $40.1 \mathrm{mmHg}$, a $\mathrm{pO}_{2}$ of $113 \mathrm{mmHg}$, and an $\mathrm{SpO}_{2}$ of $96 \%$ at an $\mathrm{FiO}_{2}$ of 0.3 . Thus, we were able to wean the patient from the ventilator after 3 days. The pneumothorax, subcutaneous emphysema, and pneumomediastinum also resolved spontaneously under oxygen therapy (Fig. 5) and the patient was discharged from the intensive care unit after 8 days.

\section{Discussion}

This case illustrates the danger of inappropriate manual ventilation of a patient exhibiting massive hemoptysis with an airway obstruction.

Manual ventilation is frequently required after tracheal intubation before a patient is connected to a ventilator, during intra-hospital transport, or because of persistent oxygen desaturation, despite an increase in the $\mathrm{FiO}_{2}$, during acute respiratory failure. Manual hyperinflation is also used to en- hance clearance of secretions and to re-expand lung regions exhibiting atelectasis.[2] However, inappropriate manual ventilation can induce high pressure within the airway, and/ or alveolar overdistention, triggering barotraumas.[3,4]

Barotrauma is defined by the presence of extra-alveolar air, and is often caused by overdistention and rupture of alveolar walls, proceeding down the pressure gradient from the air space into the bronchovascular sheath.[5] Risk factors for barotrauma include excessive peak inspiratory pressure, a high PEEP, a high transalveolar pressure, alveolar overdistention, and underlying lung injury.[6] The risk of excessive airway pressure is elevated during manual ventilation of an intubated patient because air cannot leak out.[7]

Our present case exhibited occlusion of the ETT, and both main bronchi, by blood clots; this created extra airway resistance, rendering manual ventilation more difficult, and in turn causing the ventilation to become excessive. Although we removed almost all of the blood clots using bronchoscopic forceps, some clots remained in the bronchi of the left lower lobe. These triggered atelectasis of the left lung, which rendered manual ventilation difficult.

Some clinicians can be relatively unconstrained when performing manual ventilation; work patterns differ significantly.[8] Manual lung hyperinflation was originally defined as inflation of the lungs with oxygen under manual compression to a tidal volume of $1.0 \mathrm{~L}$, requiring a peak inspiratory pressure of $20-40 \mathrm{~cm} \mathrm{H}_{2} \mathrm{O}$.[9] However, a previous study found that some therapists delivered pressures exceeding $100 \mathrm{~cm} \mathrm{H}_{2} \mathrm{O}$. Very high pressures were measured in high-resistance/low-compliance scenarios, yielding smaller tidal volumes. Notably, the breathing frequency during bag ventilation was also relatively high (25 breaths/min).[7]

Clinicians must be trained to avoid potentially deleterious ventilation patterns. The outcomes of manual ventilation depend on the skill of the practitioner. Ideally, a pressure manometer should be used in conjunction with a resuscitator bag to provide direct feedback to the clinician. Then this bag-ventilation technique can be adjusted to prevent extensive barotrauma, such as developed in our case.[10] Physicians should also practice ventilation of artificial lungs under various clinical situations to help them understand the ventilation patterns that they generate under different circumstances.[11]

In conclusion, if life-threatening hemoptysis develops, the 
possibility of airway occlusion by blood clots should always be considered and promptly managed. Manual ventilation of such cases must be accurate and stable, as extensive barotrauma can develop, caused by excessive airway pressure.

\section{ORCID}

\section{Chin Kook Rhee http://orcid.org/0000-0003-4533-7937}

\section{References}

1) Ricard JD: Manual ventilation and risk of barotrauma: primum non nocere. Respir Care 2005; 50: 338-9.

2) Denehy L: The use of manual hyperinflation in airway clearance. Eur Respir J 1999; 14: 958-65.

3) López Rodríguez A, López-Sánchez L, Julía JA: Pneumoperitoneum associated with manual ventilation using a bag-valve device. Acad Emerg Med 1995; 2: 944.

4) Kim JB, Jung HJ, Lee JM, Im KS, Kim DJ: Barotrauma developed during intra-hospital transfer -a case report-. Korean J Anesthesiol 2010; 59 Suppl: S218-21.

5) Macklin MT, Macklin CC: Malignant interstitial em- physema of the lungs and mediastinum as an important occult complication in many respiratory diseases and other conditions: an interpretation of the clinical literature in the light of laboratory experiment. Medicine 1944; 23: 281-358.

6) Gammon RB, Shin MS, Buchalter SE: Pulmonary barotrauma in mechanical ventilation. Patterns and risk factors. Chest 1992; 102: 568-72.

7) Turki M, Young MP, Wagers SS, Bates JH: Peak pressures during manual ventilation. Respir Care 2005; 50: 340-4.

8) McCarren B, Chow CM: Manual hyperinflation: a description of the technique. Aust J Physiother 1996; 42: 203-8.

9) Windsor HM, Harrison GA, Nicholson TJ: “Bag squeezing": a physiotherapeutic technique. Med J Aust 1972; 2: 829-32.

10) Redfern J, Ellis E, Holmes W: The use of a pressure manometer enhances student physiotherapists' performance during manual hyperinflation. Aust J Physiother 2001; 47: 121-31.

11) Patman $S$, Jenkins $S$, Smith $K$ : Manual hyperinflation: consistency and modification of the technique by physiotherapists. Physiother Res Int 2001; 6: 106-17. 